\title{
URBAN COMPUTING: A PRIMER
}

\author{
Matthew N. O. Sadiku, Adebowale E. Shadare, and Sarhan M. Musa \\ Roy G. Perry College of Engineering \\ Prairie View A\&M University \\ Prairie View, TX 77446
}

\begin{abstract}
The urbanization process has been accelerating worldwide, making cities becoming complex social ecosystems. Urban computing is an emerging field that seeks to solve problems in modern cities, such as traffic congestion, healthcare monitoring, air pollution, energy consumption, and public safety. Its goal is to develop computational solutions that make cities more livable, more efficient, and better positioned for the centuries ahead. This paper provides a brief introduction to urban computing.
\end{abstract}

Keywords: Urban Computing, Cyber-Physical Systems, Internet Of Things, Smartphones, Ubiquitous/Pervasive Computing.

\section{INTRODUCTION}

Urbanization is increasing at a rapid pace in almost all countries. Roughly half the world's population lives in urban environments. Rapid urbanization has led to the expansion of large cities, modernizing people's lives and also posing big challenges, such as air pollution, energy consumption, traffic congestion, and environment monitoring. Some of these challenges are shown in Figure 1 [1]. Urbanization is coupled with the increasing deployment of ubiquitous high-speed wireless access networks, powerful mobile devices, and large public displays which alter the ways people behave in urban spaces.

In modern cities, intelligent devices and sensor networks generate massive data. The urban big data are fed into data centers and various processes such as video compression and analysis are carried out. Data centers can carry out urban computing with these data to make various conclusions or predictions. These data play a significant role in urban management, security, and activities.

\section{CONCEPT OF URBAN COMPUTING}

From smart phone that help locate places to sensor-equipped cameras that detect traffic violations, computing is all around us in modern cities. Urban computing is the branch of pervasive computing that deals with urban settings and everyday lifestyles. The term "urban computing" was first introduced by Eric Paulos at the UbiComp conference in 2004. Urban Computing (UrC) is essentially an extension of ubiquitous computing (UC). Just like ubiquitous computing, urban computing enables users to access networked services and resources anytime, anywhere. Thus, urban computing may be regarded as computing technology that is applied to public area (such as street, park, sports stadium, train station, and school) in order to provide people with ubiquitous computing services [2].

Urban computing is the process of acquisition, integration, and analysis of big data generated by a diversity of sources in cities to create solutions which improve urban environment, human life quality, and city operation. It is an inter-disciplinary field where computer science meets urban planning, urban economy, transportation, economy, sociology, environmental science, anthropology, healthcare, and energy [3]. It uses wireless and sensor networks, information science, and human-computer interaction to enhance the quality of densely populated areas, tackle the major issues faced by modern cities, and improve the intelligence of the cities.

Technologies that enable urban computing include [4]: (1) Urban sensing, which collects data from different sources through sensors; (2) Urban data management, which manages large scale, dynamic, and heterogeneous urban data; (3) Urban data analytics, which applies data mining models and machine learning algorithms to unlock the power of knowledge from data; and (4) service providing, which offers interface that allows domain systems to call the knowledge from an urban computing application.

\section{URBAN COMPUTING FRAMEWORK}

Figure 2 illustrates a general framework of urban computing [5]. Urban computing systems combine three interrelated components: space, people, and technology or place, community and infrastructure, as shown in Figure 3[6]. The three components are explained as follows [7]:

- Place: This consists of rooms, buildings, offices, etc. Cities reflect how we see our world. Fifty percent of people around the world live in urban areas. 
- Community: This comprises of individuals and groups forming a society. We need to view cities as culturally and historically specific, rather than buildings and infrastructure.

- Infrastructure: This includes roads, transport, computer networks, etc. The networked infrastructures enable a heterogeneous and dynamic experience of the city. Connectivity is the most fundamental need of a smart city.

Because of these three elements, a deeper understanding of the existing social, cultural, and political contexts is required to build deployments that respect and enhance the experience of living a technologically mediated life [8].

Companies such as IBM, Cisco, and Hitachi are developing hardware and software packages targeted for the urban market. Urban operating systems (Urban OS), developed by these IT companies for the urban market, is transforming the way in which cities are imagined and configured while making the cities more efficient and sustainable [9]

\section{APPLICATIONS}

Some of the applications of urban computing are shown in Figure 4 [10] and explained as follows [11-13]:

- Transportation: This is one of the major applications of urban computing. Urban computing can cheaply improve private and public transportation in a given city. It can aid making decisions on transportation policy. It can help select better driving routes. To enable efficient taxi dispatch and monitoring, taxis are equipped with GPS sensors, Uber is an on-demand taxi service where users can request rides with their cell phone. Urban computing is an integral part of Uber's vision to improve the health, environment, and safety of cities and their communities.

- Pollution: Air pollution in an urban area can be predicted based on the data collected from air pollution monitoring stations. Urban computing can be used to track and predict pollution in some areas and prevent the negative health effects.

- Social Interaction: Urban computing enables digitally facilitated interaction. Mobile computing devices can be used to facilitate social interaction. World Health Organization (WHO) has used social media platforms to provide rapid dissemination of news such as disease outbreaks and medical discoveries.

- Environment: This is an important topic in urban computing. Urban computing can improve the environment people live in, such as by raising citywide air quality and reducing noise pollution. Urban computing considers the use of ubiquitous computing technologies in urban environments.

- Urban Planning: This is one of the most significant applications of urban computing. There is the need for urban planning that combines land use planning with transportation planning in order to improve the economic and social environments of urban dwellers.

- Urban Agriculture: This involves growing plants, raising animals, and distributing food products in the urban spaces. Urban agriculture is often performed in small-scale in small areas like backyards, terraces, rooftops, patios, along rivers, roads, and railways, or under power lines.

\section{BENEFITS AND CHALLENGES}

Urban computing tackles urban challenges such as air pollution, environmental pollution, ever-increasing energy consumption, and traffic congestion. It is useful for policy making, real-estate development, and surveillance. It improves people's life quality and helps build smarter cities. It promotes economic development and digital inclusion. It can help implement value-added applications in the smart city. It also helps us understand the nature of urban phenomena and even predict the future of cities. Urban computing has touched the three major elements of urban life: place, community and infrastructure.

Urban computing presents challenges to the design of technology within the social complexities of public space. Due to the increasing heterogeneity, complexity and large volumes of urban data, analyzing them is challenging. It calls for more advanced data analysis technologies and often requires integrating human perception in analytical process and a broad use of visualization. Urban data of new types keep emerging, most of which cannot be visualized using existing methods [14]. A major bottleneck in data centers for urban computing is limited memory size. In spite of the challenges, urban computing is, to a limited extent, already a mass phenomenon.

\section{CONCLUSION}

The modern city is currently undergoing a paradigm shift, where shared public spaces are becoming increasingly saturated with new types of pervasive computing technology. Urban computing is an emerging field that deals with how people experience the smart cities with the support of technologies. Technology designers should consider becoming urban planners and social scientists so that they may create technologies that can augment social life in positive ways.

Urban Computing Foundation (UCF) is an organization that is dedicated to accelerating the open development of software that improves urban mobility, transportation, and safety. Uber and Facebook are strong members of UCF. The aim of UCF is to find better methods to interpret and transmit the data in a world where people and machines are more interconnected. More information about urban computing can be obtained from the books in [15-18]. 


\section{REFERENCES}

[1] Y. Zheng, "Urban computing -Using big data to solve urban challenges," unknown source.

[2] H. Ko et al., "Contexts-management strategy with security consideration in urban computing based on urban design," Proceedings of the Fifth International Conference on Innovative Mobile and Internet Services in Ubiquitous Computing, 2011, pp. 65-72.

[3] Y. Zheng, "Urban computing: Tackling urban challenges using big data," Proceedings of IEEE 24th International Requirements Engineering Conference, Beijing, China, 2016.

[4] Y. Zheng, "Urban computing: Enabling urban intelligence with big data,"

Frontiers of Computer Science, vol. 11, no. 1, February 2017, pp. 1-3.

[5] Y. Zheng, "Urban computing: Concepts, methodologies, and applications,"

ACM Transaction on Intelligent Systems and Technology, October 2014.

[6] D. Ringas and E. Christopoulou, "Collective city memory: Field experience on the effect of urban computing on community," Proceedings of the 6th International Conference on Communities and Technologies, Munich, Germany, June- July, 2013, pp. 157-165.

[7] A. Williams and P. Dourish, "Imagining the city: The cultural dimensions of urban computing," Computer, September 2006, pp. 38-43.

[8] H. Kukka et al., "Urban computing in theory and practice: Towards a transdisciplinary approach," Nordic Conference on Human-Computer Interaction, Helsinki, Finland, October 2014, pp. 658-667.

[9] S. Marvin and A. Luque-Ayala, "Urban operating systems: Diagramming the city," International Journal of Urban and Regional Research, vol. 41, no. 1, June 2017, pp. 84-103.

[10] C. Kamienski, "Urban computing technologies and applications for smart cities,"

https://www.researchgate.net/publication/316438664_Urban_Computing_Technologies_and_Applications_for_Smart_Cities/link/ 59004e8a0f7e9bcf65456c66/download

[1] "Urban computing," Wikipedia, the free encyclopedia

https://en.wikipedia.org/wiki/Urban_computing

[12] Y. Zheng et al., "Urban computing with taxicabs," Proceedings of the 13th international conference on Ubiquitous computing, Beijing, China, 2011, pp. 89-98.

[13] S. Serra, P. V. Cruz, and C. M. Vieira, "Vision paper: Challenges and opportunities of social computing in urban agriculture in global north and south countries,"

https://www.semanticscholar.org/paper/Vision-Paper\%3A-Challenges-and-Opportunities-of-in-in-Serra-

Cruz/1be9d896f37b873a9f678803125b818da449b426

[14] Y. Zheng et al., "Visual analytics in urban computing: An overview," IEEE Transactions on Big Data, vol. 2, no. 3, JulySeptember 2016, pp. 276-296.

[15] Y. Zheng, Urban computing. MIT Press, 2019.

[16] T. Kim, A. Stoica, and R. S. Chang (eds.), Security-Enriched Urban Computing and Smart Grid. Berlin, Germany: SpringerVerlag, 2010.

[17] A. F. Loureiro and H. Silva (eds.), Urban Computing. Springer, 2019.

[18] J. Oliveira et al (eds.), Big Social Data and Urban Computing. Springer, 2019.

\section{AUTHORS}

Matthew N.O. Sadiku is a professor at Prairie View A\&M University, Texas. He is the author of several books and papers. He is an IEEE fellow. His research interests include computational electromagnetics and computer networks.

Adebowale Shadare just completed his doctoral programt at Prairie View A\&M University, Texas. He is the author of several papers.

Sarhan M. Musa is a professor in the Department of Electrical and Computer Engineering at Prairie View A\&M University, Texas. He has been the director of Prairie View Networking Academy, Texas, since 2004. He is an LTD Sprint and Boeing Welliver Fellow. His research interests include computer networks and computational electromagnetics. 
International Journal of Advances in Scientific Research and Engineering (ijasre), Vol 5 (10), October-2019
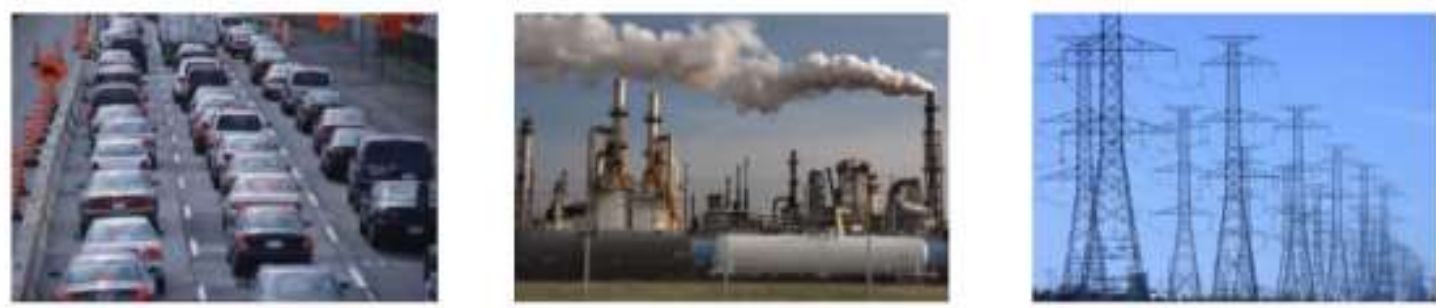

Figure 1 Some urban challenges [1].

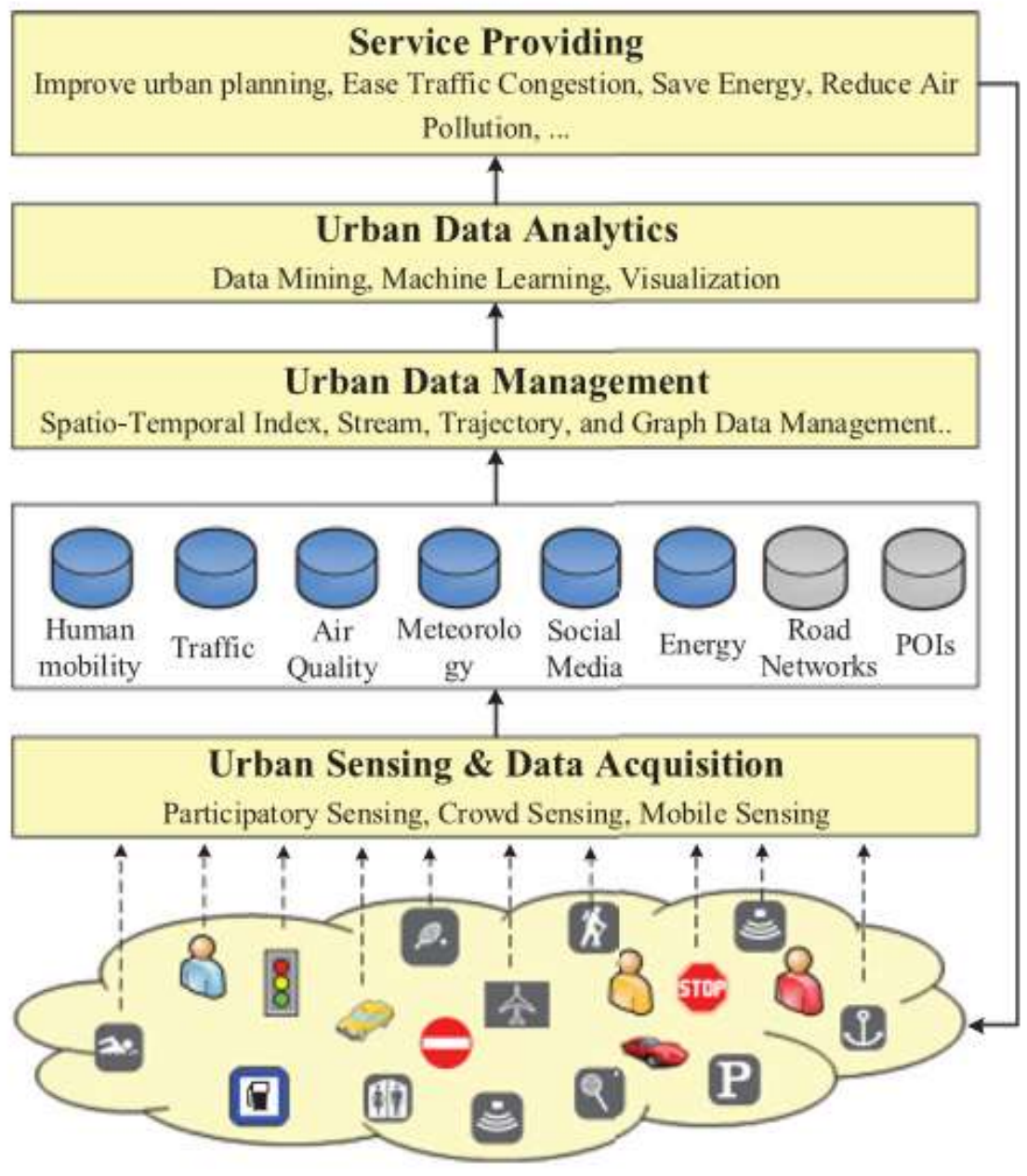

Figure 2 A general framework of urban computing [5]. 


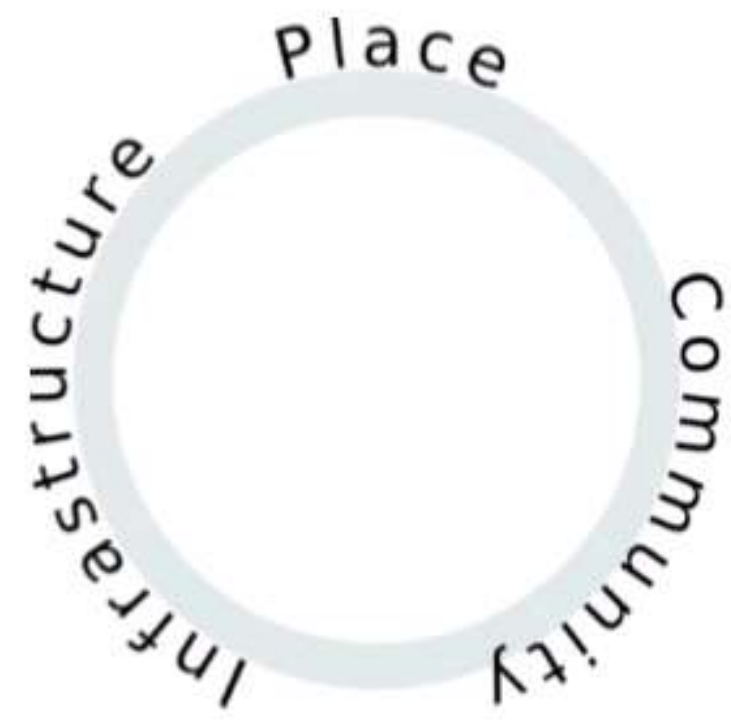

Figure 3 The three urban computing components [6].

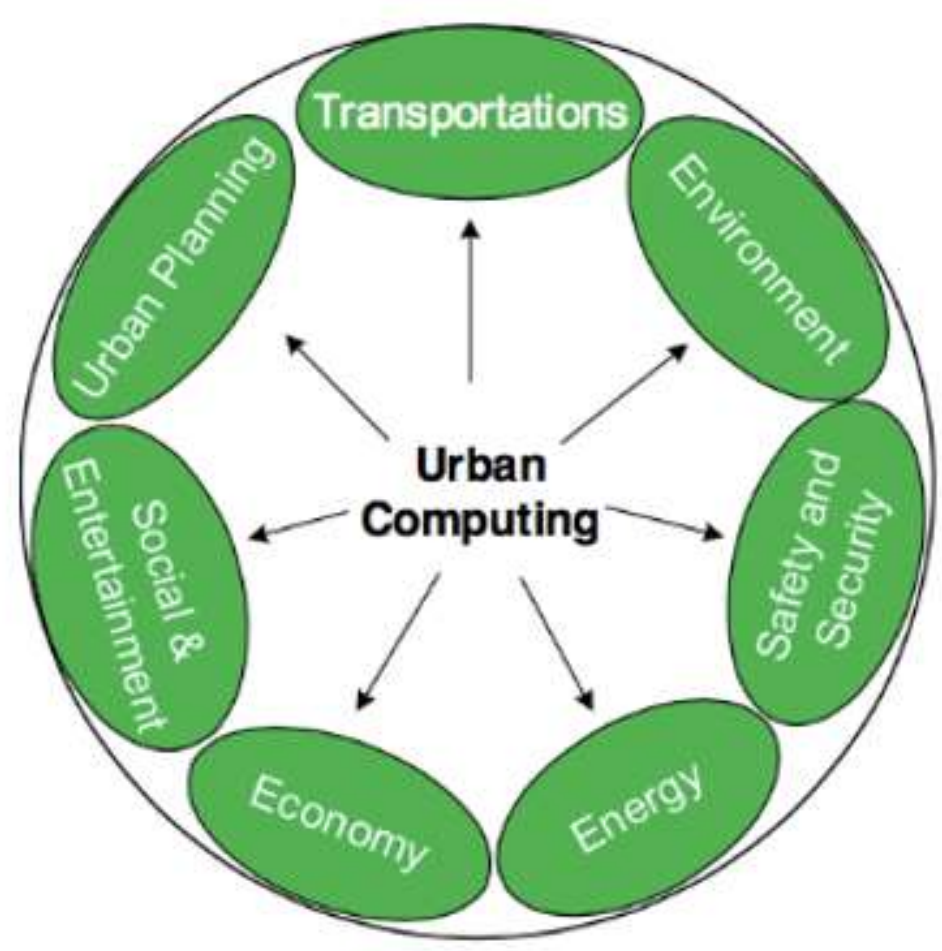

Figure 4 Some of the applications of urban computing [10]. 